\title{
Improving Data Analysis using Data Mining Techniques for KSOMM and PAPLM
}

\author{
Vivek Rajput ${ }^{1}$, Prof.Amit Thakur ${ }^{2}$ \\ M. Tech., Scholar, Department of CSE, SVCST, RGPV, Bhopal, India ${ }^{1}$ \\ Assistant Professor, Department of CSE, SVCST, RGPV, Bhopal, India ${ }^{2}$
}

\begin{abstract}
Cluster analysis could also be a descriptive task that seeks to identify consistent cluster of object and it's in addition one in all the foremost analytical technique in processing. K-means cluster with self organization map methodology a neural network methodology is that the popular divided bunch technique. They need a tendency to debate commonplace $\mathrm{k}$ mean formula and analyze the defect of $\mathrm{k}$-mean formula. Throughout this paper three dissimilar modified $\mathrm{k}$-mean formulas are mentioned that exclude the limitation of $\mathrm{k}$-mean formula and improve the speed and efficiency of k-mean formula. Experiments supported the quality information UCI show that the projected technique will find yourself a high purity cluster results and eliminate the sensitivity to the initial centers to some extent. E.Coli dataset and Yeast dataset resides issue organism and altogether all completely different super molecule assign in their cell. If that protein is wounded, then these cause varied infections that affected anatomy adversely. So, the target of this work is to classify proteins into altogether all completely different cellular localization sites supported chemical compound sequences of E.Coli bacteria and Yeast. It's found that projected bunch provides correct result as compared to K-Mean and is ideal resolution to localization of proteins. It's in addition known as nearest neighbor trying. It simply clusters the datasets into given kind of clusters. Varied efforts are created to boost the presentation of the K-means bunch formula and our Planned Advanced Proteins Localization Methodology (PAPLM) in rising accuracy of information proteins level analysis then notice best answer. They need mentioned the restrictions and applications of the K-means bunch formula still. Discover our projected formula best resolution. Improving information analysis victimization data processing techniques for KSOMM and PAPLM
\end{abstract}

Keywords: Data Processing, Cluster Technique, Hierarchical Cluster, K-Mean Cluster, Performance Accuracy, Optimization Algorithmic Rule

\section{INTRODUCTION}

Clustering might be a technique of grouping data objects into disjointed clusters that the information inside an equivalent cluster are similar, but data happiness to fully take issue completely different cluster differ. A cluster is collections of data object that are nearly like different are in same cluster and dissimilar to the objects area unit in different clusters. The demand for organizing the sharp increasing data and learning valuable information from data, that creates bunch techniques area unit wide applied in many application areas like computing, biology, consumer relationship management, data compression, processing, information retrieval, image process, machine learning, marketing, medicine, pattern recognition, psychology, statistics thus on. Cluster analysis may be a tool that is accustomed observes the characteristics of cluster and to concentrate on a selected cluster for a lot of analysis. Bunch is unsupervised learning and do not trust predefined classes. In bunch they have a tendency to measure the distinction between objects by measure the area between each strive of objects. These live embrace the geometrician, Manhattan and Hermann Murkowski Distance. The terms processing, patent mining, text mining and image square measure used for the method of the documents. This chapter will try to give some explanations of the terms and justify data mining was chosen for the title of the study. data processing is that the analysis of (often large) experimental data sets to search out unsuspected relationships and to summarize the info in novel ways in which are each intelligible and helpful to the info owner. Bunch could be a division of information into teams of comparable objects. Representing the info by fewer clusters essentially loses bound fine details, however achieves simplification. It models information by its clusters. Information modeling puts bunch in an exceedingly historical perspective rooted in arithmetic, statistics, and numerical analysis [1]. The notion of a "cluster" varies between algorithmic programs and is one in all the various choices to require once selecting the acceptable algorithm for a selected drawback. Initially the nomenclature of a cluster looks obvious: a bunch of information objects. However, the clusters found by totally different algorithms vary considerably in their material goods and considerate these bunch representation are significant to considerate the variations in the numerous of types algorithms. usual grouping representation contain: material goods representation, midpoint of group representation, allocation representation, thickness representation space representation, cluster representation and Graph-based representation [2,3, and 4]. 


\section{CENTROID-AGGLOMERATION AND PARTITION-AGGLOMERATION}

In centroid-agglomeration and partition-agglomeration and clusters unit of dimension outline by a middle vector point that cannot fundamentally be a component of the data or information.

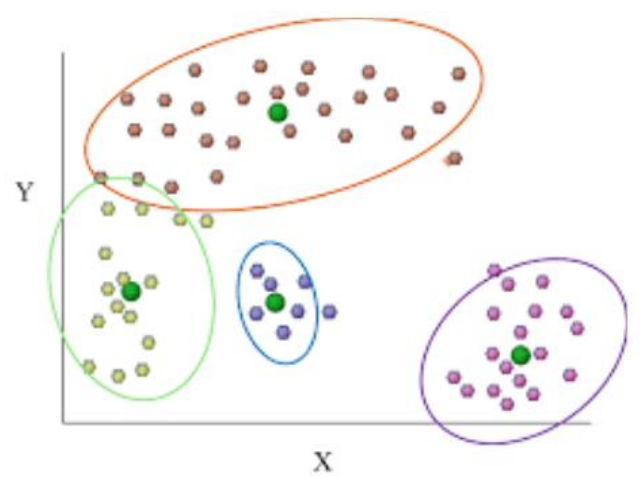

Fig1 Different Clustering

K-means clustering method is a way of clustering that's broadly used. This set of rules is the maximum famous clustering device this is utilized in clinical and business packages. it's far a way of cluster analysis which targets to partition observations into okay clusters in which every statement belongs to the cluster with the nearest imply is one of the most extensively used clustering algorithms. The algorithm walls the data points into c companies in an effort to reduce the sum of the distances between the statistics points and the middle of the clusters. Regardless of it simplicity, the ok-means set of rules entails a very large quantity of nearest neighbor queries. The high time complexity of the kway set of rules makes it impractical for use within the case of having a huge range of factors in the records set. Lowering the massive variety of nearest neighbor queries inside the algorithm can boost up it. In addition, the range of distance calculations will increase exponentially with the increase of the dimensionality of the records] to cluster user queries. However, in that work, best consumer clicks have been used. In our technique, we combine each user clicks and report and question contents to determine the similarity. Better outcomes [5].

\section{LITERATURE SURVEY}

[6] has provided the results of the effect of skewed data distribution on K-means clustering. They have given an organized study of K-means and cluster validation measures from a data distribution perspective. In fact, addition to entropy measure and f-measure.

[7] The data points are then divided into k cluster i.e. number of desired cluster. Finally the nearest possible data point of the mean is taken as initial centroid. Experimental outputs show that the algorithm reduces the number of iterations to assign data into a cluster. But the algorithm still deals with the problem of assigning number of desired cluster as input.

[8] Discussed different methodologies and parameters associated with different clustering algorithms. They also discussed on issues in different clustering algorithms used in large datasets.

[9]Worked out an adaptive particle swarm optimization (PSO) on individual level. By analyzing the social model of PSO, a replacing criterion based on the diversity of fitness between current particle and the best historical experience is introduced to maintain the social attribution of swarm adaptively by removing inactive particles. Three benchmark functions were tested which indicates its improvement in the average performance

[12] A proposed a title "An Improved innovative Center Using K-means Clustering Algorithm and FCM"by the problem of random selection of initial centroid and similarity measures, the researcher presented a new K-means clustering algorithm based on dissimilarity (Axiomatic Fuzzy Sets) topology neighborhoods' are employed to determine the clustering initial points. The AFS global k-means algorithms are introduced, in which the distance based on the AFS topology neighborhood is employed in the step of determining initial cluster centers.

[13] Have presented order constrained solution in K-means as a more stable method for clustering of sound features.

[14] Recently, a self-organizing multi objective evolutionary algorithm was evaluated on some state-of-the-art multi objective evolutionary methods. A local PCA partitions the given population into several disjointed clusters, and conducts PCA in each cluster to extract a continuous manifold and build a probabilistic model.

[15] Have improved the traditional K-means algorithm by making analysis on the statistical data 
Vol. 8, Issue 6, June 2019

[16] .In clustering easy k-means and Genetic algorithm. method is combine with GA to get the optimize no. of clusters from the result of simple k-mean set of rules .both algorithm are simple to understand and may be relevant for numerous form of facts like genomic data set, numerical dataset.

\section{SIMULATION TOOL}

MAT-LAB tool may be a collection of tool and performance for top performance mathematical computation and visual image .MATLAB (2013a) is that the high level language and interactive setting utilized by several engineers and scientists worldwide. It lets the explore and visualize concepts and collaborate across totally different disciplines with signal and image process, communication and computation of results. MATLAB (2013a) provides tools to amass, analyze, and visualize information, modify you to induce insight into your information in a very division of the time it might take victimization spreadsheets or ancient programming languages. It may also document and share the results through plots and reports or as revealed MATLAB (2013a) code .MATLAB (2013a) (matrix laboratory) may be a multi paradigm numerical computing state of affairs and fourth generation artificial language. it's developed by scientific discipline work; MATLAB (2013a) permits matrix strategy, plotting of operate and information, implementation of algorithmic rule, construction of user interfaces with programs. MATLAB (2013a) is meant primarily for mathematical computing.

\section{RESULT ANALYSIS}

In the field of information mining and determine several challenge and need the challenges in dataset analysis improved accuracy and following objectives. Find dataset accuracy and its found minimize cluster size and optimum answer.

(a) E_coil information analysis using previous methodology KSOMM primarily based weight graph to represent weight graph in show below and a lot of copy information in show in graph and accuracy low. information analysis then generate totally different purpose like $n p=n p 1, n p 2 \ldots . . n p n$ with corresponding weight vector $w n=w 1, w 2 \ldots$. Wn .

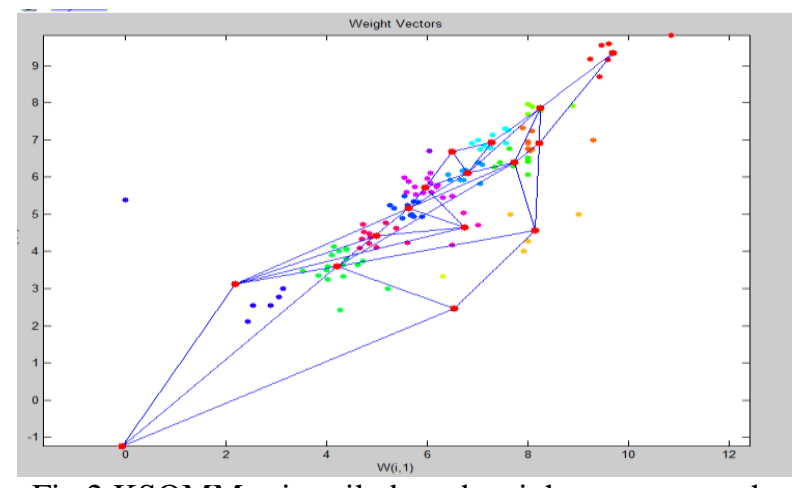

Fig.2 KSOMM primarily based weight vectors graph

(a) Planned methodology primarily based weight vectors graph: E_coil information analysis using previous methodology planned methodology primarily based weight graph to represent weight graph in show below and less copy information in show in graph and accuracy high. Information analysis then generate totally different purpose like $\mathrm{np}=\mathrm{np} 1, \mathrm{np} 2 \ldots . . \mathrm{npn}$ with corresponding weight vector $\mathrm{wn}=\mathrm{w} 1, \mathrm{w} 2 \ldots \mathrm{Wn}$.

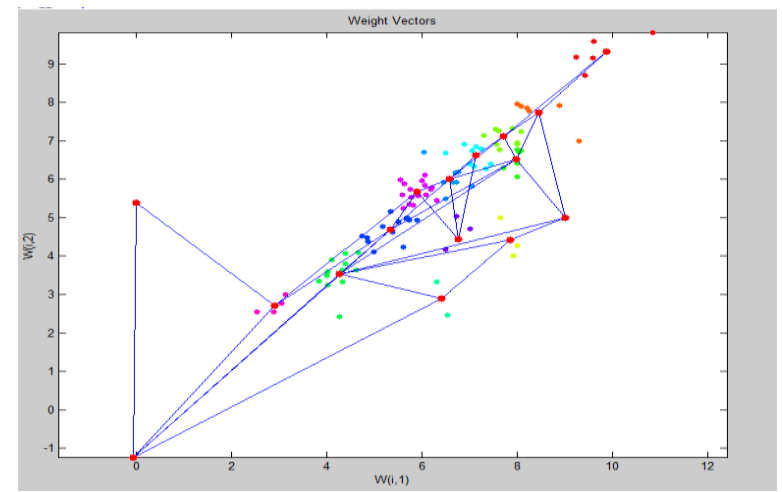

Fig.3 planned methodology primarily based weight vectors graph 
Vol. 8, Issue 6, June 2019

(c)Accuracy analysis between PAPLM and KSOMM:

Accuracy analysis finds victimization our planned methodology (PAPLM) in additional accuracy and previous methodology (KSOMM) less accuracy .in figure 5.8

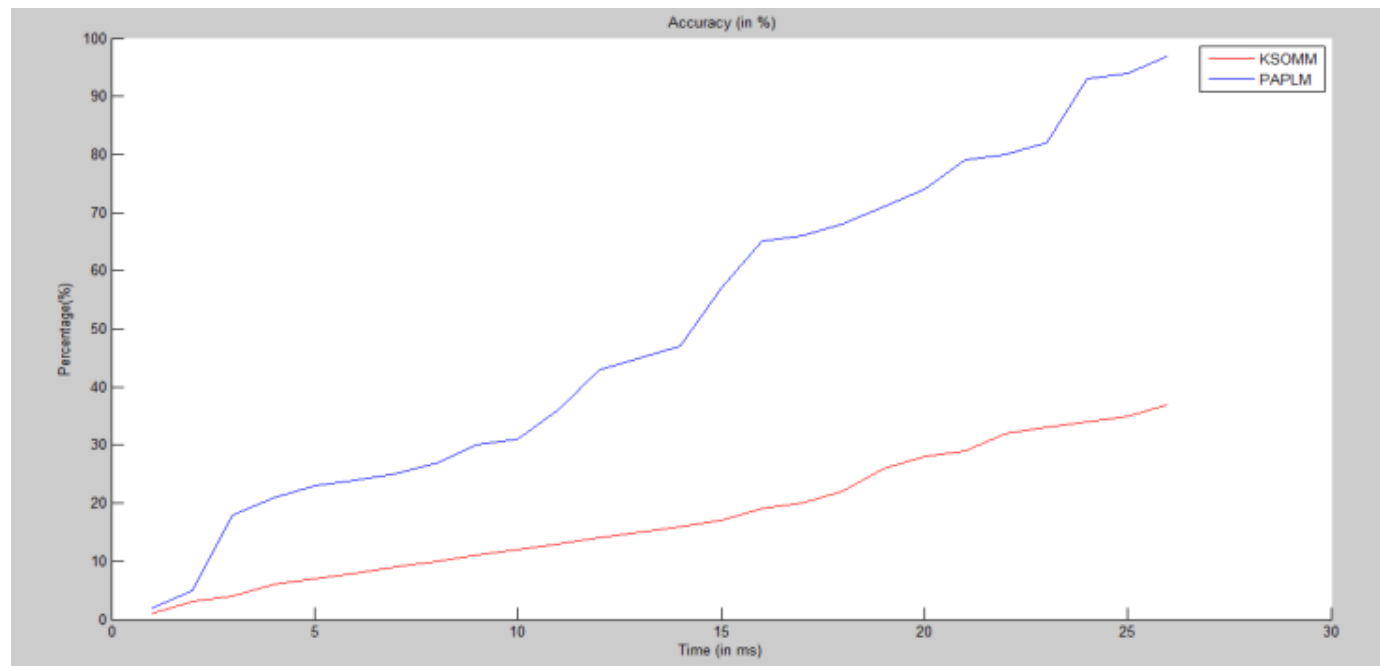

Fig.4Accuracy analysis between PAPLM and KSOMM

(d) KSOMM primarily based values graph: In show figure 5.9, KSOMM primarily based values graph analysis graph represent error is high.

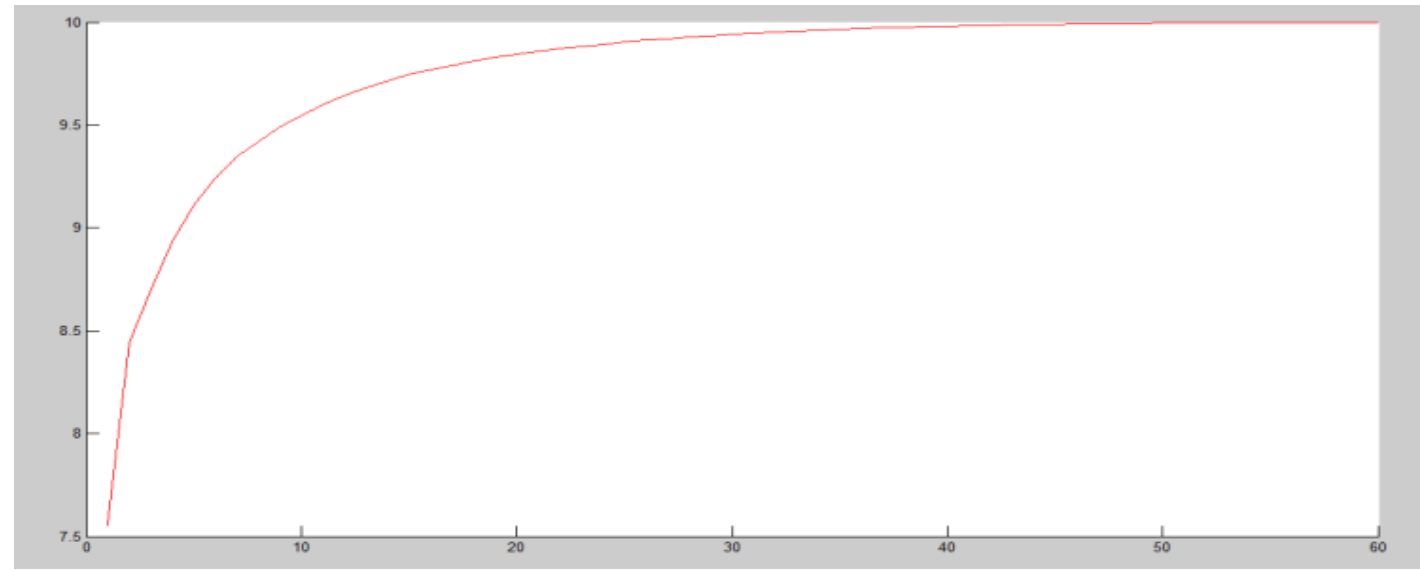

Fig.5 KSOMM primarily based values graph

(e) Planned methodology primarily based values graph: In show figure 5.10, planned methodology primarily based values graph analysis graph represent error is a smaller amount

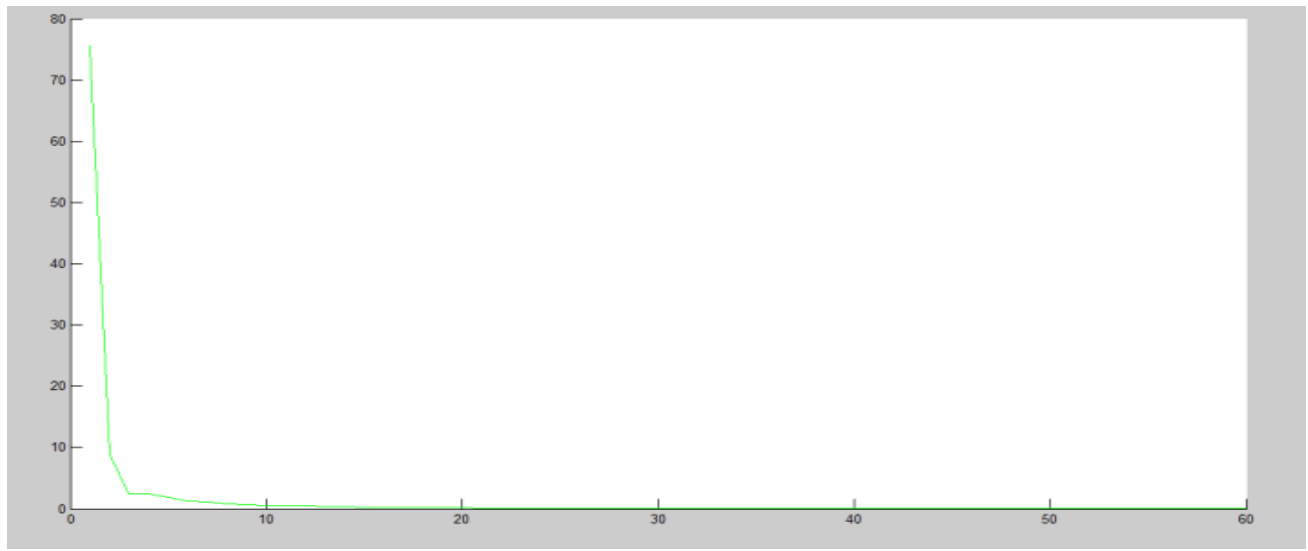

Fig6 planned methodology primarily based values graph 


\author{
Vol. 8, Issue 6, June 2019
}

\title{
VI. CONCLUSION
}

Clustering could also be a method of information mining. It's associate unattended learning technique that does not have faith in predefined model and output classes. Cluster analysis is not a one-shot technique. In many circumstances, it desires a series of trials and repetitions. Moreover, there are not any universal and effective criteria to guide the selection of choices and clump schemes. Throughout this text they've offered elaborate survey on fully totally different $\mathrm{K}$-means. To simulate associate improved innovative and optimum performance analysis supported e.coil dataset and yeast dataset victimization processing techniques to k-means cluster and projected cluster for increase the performance using E_Coil dataset and YEAST dataset. Projected techniques are performance analysis every dataset. Notice optimum result supported E_Coil dataset and yeast dataset mistreatment $\mathrm{KMC}$ and projected techniques. It's going to be found higher outcomes in cross validation and k-mean cluster as a result of extra accuracy supported base on minimizing redundancy in dataset and minimize fault. As in initial formula time complexity is greater as compared to plain k-mean formula for large data set thus it's going to be everywhere that if they need a tendency to use arrange of third formula i.e. they need a bent to use system to store information in initial algorithmic rule. They'll reduce the time complexity of that algorithmic rule and outcome in optimum answer.

\section{REFERENCES}

[1]. Nishchal K. Verma, Abhishek Roy "Self-Optimal Clustering Technique Using Optimized Threshold Function" IEEE SYSTEMS JOURNAL, IEEE 2013. Pp 1-14.

[2]. Shafiq Alam, Gillian Dobbie, Patricia Riddle, M. Asif Naeem,(2010). "Particle Swarm Optimization Based Hierarchical Agglomerative Clustering". IEEE/WIC/ACM International Conference on Web Intelligence and Intelligent Agent Technology, pp. 64-68.

[3]. Lan Yu, "Applying Clustering to Data Analysis of Physical Healthy Standard", 2010 Seventh International Conference on Fuzzy Systems and Knowledge Discovery (FSKD 2010), pp. 2766-2768

[4]. Yun Ling and Hangzhou, "Fast Co-clustering Using Matrix Decomposition", IEEE (2009). Asia-Pacific Conference on Information Processing, pp. 201-204.

[5]. Madjid Khalilian, Farsad Zamani Boroujeni, Norwati Mustapha, Md. Nasir Sulaiman,(2009). "K-Means Divide and Conquer Clustering", IEEE 2009, International Conference on Computer and Automation Engineering, pp. 306-309.

[6]. H. Xiong; J. Wu; J. Chen, "K-Means Clustering Versus Validation Measures: A Data-Distribution Perspective, " IEEE Transactions on Systems, Man, and Cybernetics, Part B: Cybernetics, vol.39, no.2, pp.318, 331, April 2009.

[7]. Zhang, X.; Tian, Y.; Cheng, R.; Jin, Y. A Decision Variable Clustering-Based Evolutionary Algorithm for Large-scale Many-objective Optimization. IEEE Trans. Evolut. Comput. 2016.

[8]. M. Vijayalakshmi, M.R. Devi, “ A Survey of Different Issue of Different clustering Algorithms Used in Large Data sets”, International Journal of Advanced Research in Computer Science and Software Engineering, Volume 2, Issue 3, March 2012 ISSN: 2277 128X.

[9]. Kennedy, J. Stereotyping: Improving particle swarm performance with cluster analysis. In Proceedings of the IEEE Congress on Evolutionary Computation, La Jolla, CA, USA, 16-19 July 2000; pp. 1507-1512.

[10]. Bagirov, Adil M. "Modified global k-means algorithm for minimum sum-of-squares clustering problems." Pattern Recognition 41, no. 10 (2008): 3192-3199.

[11]. Wang, Lidong, Xiaodong Liu, and Yashuang Mu. "The Global k-Means Clustering Analysis Based on Multi-Granulations Nearness Neighborhood." Mathematics in computer science 7, no. 1 (2013): 113-124.

[12]. S. Krey, U. Ligges, F. Leisch, "Music and timbre segmentation by recursive constrained K-means clustering", Computational Statistics, February 2014, Volume 29, Issue 1-2, pp 37-50

[13]. Zhang, H.; Zhou, A.; Song, S.; Zhang, Q.; Gao, X.-Z.; Zhang, J. A self-organizing multiobjective evolutionary algorithm. IEEE Trans. Evolut. Comput. 2016, 20, 792-806.

[14]. H. Xiuchang, SU Wei, “An Improved K-means Clustering Algorithm”, JOURNAL OF NETWORKS, vol. 9, No. 1, Jan 2014.

[15]. Zhang, J.; Chung, H.S.-H.; Lo, W.-L. Clustering-based adaptive crossover and mutation probabilities for genetic algorithms. IEEE Trans. Evolut. Comput. 2007, 11, 326-335.

[16]. Madhulatha, T. Soni. "An overview on clustering methods." arXiv preprint arXiv:1205.1117 (2012).

[17]. Karypis, George, Eui-Hong Sam Han, \& Vipin Kumar "Chameleon: Hierarchical clustering using dynamic modeling."Computer 8(1999):68-75.

[18]. Zhang, Tian, Raghu Ramakrishnan, and Miron Livny. "BIRCH: A new data clustering algorithm and its applications." Data Mining and Knowledge Discovery 1, no. 2 (1997): 141-182.

[19]. Popat, Shraddha K., and M. Emmanuel. "Review and comparative study of clustering techniques." International journal of computer science and information technologies 5.1 (2014): 805-812.

[20]. Berkhin, Pavel. "A survey of clustering data mining tech." In Grouping multidimensional data, pp.25-71. Springer, Berlin, Heidelberg, 2006.

[21]. Vesanto, Juha, and Esa Alhoniemi. "Clustering of the self-organizing map." IEEE Transactions on neural networks 11, no. 3 (2000): 586-600.

[22]. Lung, Chung-Horng, Marzia Zaman, and Amit Nandi. "Applications of clustering techniques to software partitioning, recovery and restructuring." Journal of Systems and Software73, no. 2 (2004): 227-244.

[23]. Xing, Eric P., Michael I. Jordan, Stuart J. Russell, and Andrew Y. Ng. "Distance metric learning with application to clustering with sideinformation." In Advances in neural information processing systems, pp. 521-528. 2003.

[24]. Horton, Paul, and Kenta Nakai. "Better Prediction of Protein Cellular Localization Sites with the it k Nearest Neighbors Classifier." In Ismb, vol. 5, pp. 147-152. 1997 